\title{
Adsorption of 5-Hydroxymethylfurfural, Levulinic Acid, Formic Acid and Glucose Using Polymeric Resins Modified with Different Functional Groups
}

Lei Hua,"\#, Jiayi Zheng, ${ }^{\mathrm{a}, \#}$, Qing Li ${ }^{\text {a }}$, Shunhui Tao ${ }^{\mathrm{a}}$, Xiaojie Zheng ${ }^{\mathrm{a}}$, Xiaodong Zhang,

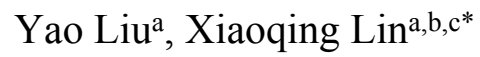

a School of Chemical Engineering and Light Industry, Guangdong University of Technology, No. 100 Waihuan Xi Road, Panyu District, Guangzhou 510006, People’s Republic of China

${ }^{\mathrm{b}}$ Guangdong Key Laboratory of Plant Resources Biorefinery, Guangdong University of Technology, Guangzhou, 510006, People's Republic of China

${ }^{c}$ Guangzhou Key Laboratory of Clean Transportation Energy Chemistry, Guangdong University of Technology, Guangzhou, 510006, People's Republic of China

\# These authors contributed equally to this study.

* Corresponding author: Prof. Xiaoqing Lin (X. Lin)

E-mail:linxiaoqing@gdut.edu.cn 
Table S1 Adsorption capacity of 5-HMF (5.021 $\left.\mathrm{g} \mathrm{L}^{-1}\right)$, LA (20.251 $\left.\mathrm{g} \mathrm{L}^{-1}\right)$, FA $\left(9.103 \mathrm{~g} \mathrm{~L}^{-1}\right)$ and glucose $\left(9.854 \mathrm{~g} \mathrm{~L}^{-1}\right)$ onto XAD-4, XAD7HP and XAD761 resins in multi-component system at $298 \pm 1 \mathrm{~K}$.

\begin{tabular}{|c|c|c|c|c|c|c|c|c|}
\hline \multirow{3}{*}{ Resin } & \multicolumn{8}{|c|}{ Component } \\
\hline & \multicolumn{2}{|c|}{ Glucose } & \multicolumn{2}{|c|}{ FA } & \multicolumn{2}{|c|}{ LA } & \multicolumn{2}{|c|}{ 5-HMF } \\
\hline & $\begin{array}{c}q_{t} \\
(\mathrm{mg} / \mathrm{g})\end{array}$ & STDV & $\begin{array}{c}q_{t} \\
(\mathrm{mg} / \mathrm{g})\end{array}$ & STDV & $\begin{array}{c}q_{t} \\
(\mathrm{mg} / \mathrm{g})\end{array}$ & STDV & $\begin{array}{c}q_{t} \\
(\mathrm{mg} / \mathrm{g})\end{array}$ & STDV \\
\hline XAD-4 & 6.2 & 1.70 & 8.2 & 0.9 & 40.0 & 4.46 & 24.3 & 0.87 \\
\hline XAD7HP & 5.4 & 1.57 & 7.2 & 0.61 & 35.6 & 2.60 & 13.8 & 0.38 \\
\hline XAD761 & 3.2 & 0.45 & 6.8 & 1.10 & 83.8 & 0.83 & 53.4 & 1.01 \\
\hline
\end{tabular}


Table S2 Adsorption selectivity of 5-HMF (5.021 $\left.\mathrm{g} \mathrm{L}^{-1}\right)$, LA (20.251 $\left.\mathrm{g} \mathrm{L}^{-1}\right)$, FA $\left(9.103 \mathrm{~g} \mathrm{~L}^{-1}\right)$ and glucose $\left(9.854 \mathrm{~g} \mathrm{~L}^{-1}\right)$ onto XAD-4, XAD7HP and XAD761 resins in multi-component system at $298 \pm 1 \mathrm{~K}$.

\begin{tabular}{ccccccc}
\hline \multirow{2}{*}{ Resin } & \multicolumn{7}{c}{ Selectivity factor } \\
& $a_{G \text { Glucoe }}^{5-H M F}$ & STDV & $a_{F A}^{5-H M F}$ & STDV & $a_{L A}^{5-H M F}$ & STDV \\
\hline XAD-4 & 8.86 & 2.52 & 5.88 & 0.53 & 2.63 & 0.23 \\
XAD7HP & 5.53 & 1.55 & 3.61 & 0.05 & 1.60 & 0.17 \\
XAD761 & 42.42 & 5.84 & 18.41 & 0.50 & 3.01 & 0.10 \\
\hline
\end{tabular}


Table S3 5-HMF adsorption capacity on XAD-4 resin in single-component system at various temperatures.

\begin{tabular}{|c|c|c|c|c|c|c|c|c|c|c|c|}
\hline \multicolumn{12}{|c|}{ Temperature } \\
\hline \multicolumn{3}{|c|}{$288 \mathrm{~K}$} & \multicolumn{3}{|c|}{$298 \mathrm{~K}$} & \multicolumn{3}{|c|}{$308 \mathrm{~K}$} & \multicolumn{3}{|c|}{$318 \mathrm{~K}$} \\
\hline $\begin{array}{c}c_{e} \\
(\mathrm{~g} / \mathrm{L})\end{array}$ & $\begin{array}{c}q_{e} \\
(\mathrm{mg} / \mathrm{g})\end{array}$ & STDV & $\begin{array}{c}c_{e} \\
(\mathrm{~g} / \mathrm{L})\end{array}$ & $\begin{array}{c}q_{e} \\
(\mathrm{mg} / \mathrm{g})\end{array}$ & STDV & $\begin{array}{c}c_{e} \\
(\mathrm{~g} / \mathrm{L})\end{array}$ & $\begin{array}{c}q_{e} \\
(\mathrm{mg} / \mathrm{g})\end{array}$ & STDV & $\begin{array}{c}c_{e} \\
(\mathrm{~g} / \mathrm{L})\end{array}$ & $\begin{array}{c}q_{e} \\
(\mathrm{mg} / \mathrm{g})\end{array}$ & STDV \\
\hline 0.00 & 0.0 & 0.00 & 0.00 & 0.0 & 0.00 & 0.00 & 0.0 & 0.00 & 0.00 & 0.0 & 0.00 \\
\hline 0.37 & 6.6 & 0.30 & 0.39 & 6.0 & 0.28 & 0.41 & 5.1 & 0.23 & 0.42 & 4.7 & 0.21 \\
\hline 0.82 & 11.1 & 0.52 & 0.85 & 9.5 & 0.39 & 0.87 & 8.5 & 0.37 & 0.87 & 8.2 & 0.31 \\
\hline 1.23 & 14.1 & 0.60 & 1.25 & 13.3 & 0.57 & 1.28 & 11.7 & 0.49 & 1.30 & 10.2 & 0.46 \\
\hline 1.68 & 17.4 & 0.78 & 1.73 & 15.4 & 0.62 & 1.76 & 13.7 & 0.58 & 1.77 & 13.4 & 0.55 \\
\hline 2.16 & 19.2 & 0.73 & 2.21 & 17.2 & 0.62 & 2.22 & 17.2 & 0.81 & 2.24 & 15.5 & 0.60 \\
\hline 2.61 & 22.4 & 0.72 & 2.68 & 20.0 & 0.92 & 2.69 & 18.9 & 0.78 & 2.71 & 18.0 & 0.85 \\
\hline 3.49 & 26.4 & 1.30 & 3.56 & 23.1 & 0.81 & 3.59 & 22.4 & 0.96 & 3.61 & 21.4 & 0.90 \\
\hline 4.46 & 29.2 & 1.23 & 4.51 & 27.2 & 1.31 & 4.53 & 25.7 & 1.13 & 4.57 & 24.1 & 0.99 \\
\hline
\end{tabular}


Table S4 5-HMF adsorption capacity on XAD7HP resin in single-component system at various temperatures.

\begin{tabular}{|c|c|c|c|c|c|c|c|c|c|c|c|}
\hline \multicolumn{12}{|c|}{ Temperature } \\
\hline \multicolumn{3}{|c|}{$288 \mathrm{~K}$} & \multicolumn{3}{|c|}{$298 \mathrm{~K}$} & \multicolumn{3}{|c|}{$308 \mathrm{~K}$} & \multicolumn{3}{|c|}{$318 \mathrm{~K}$} \\
\hline $\begin{array}{c}c_{e} \\
(\mathrm{~g} / \mathrm{L})\end{array}$ & $\begin{array}{c}q_{e} \\
(\mathrm{mg} / \mathrm{g})\end{array}$ & STDV & $\begin{array}{c}c_{e} \\
(\mathrm{~g} / \mathrm{L})\end{array}$ & $\begin{array}{c}q_{e} \\
(\mathrm{mg} / \mathrm{g})\end{array}$ & STDV & $\begin{array}{c}c_{e} \\
(\mathrm{~g} / \mathrm{L})\end{array}$ & $\begin{array}{c}q_{e} \\
(\mathrm{mg} / \mathrm{g})\end{array}$ & STDV & $\begin{array}{c}c_{e} \\
(\mathrm{~g} / \mathrm{L})\end{array}$ & $\begin{array}{c}q_{e} \\
(\mathrm{mg} / \mathrm{g})\end{array}$ & STDV \\
\hline 0.00 & 0.0 & 0.00 & 0.00 & 0.0 & 0.00 & 0.00 & 0.0 & 0.00 & 0.00 & 0.0 & 0.00 \\
\hline 0.43 & 3.7 & 0.17 & 0.44 & 3.6 & 0.17 & 0.45 & 2.9 & 0.13 & 0.45 & 2.9 & 0.13 \\
\hline 0.91 & 6.5 & 0.28 & 0.92 & 5.8 & 0.28 & 0.93 & 5.2 & 0.22 & 0.93 & 5.2 & 0.24 \\
\hline 1.34 & 8.7 & 0.35 & 1.35 & 8.2 & 0.35 & 1.37 & 7.1 & 0.31 & 1.36 & 7.6 & 0.32 \\
\hline 1.81 & 11.2 & 0.40 & 1.85 & 9.7 & 0.40 & 1.85 & 8.9 & 0.37 & 1.87 & 8.5 & 0.39 \\
\hline 2.33 & 11.4 & 0.45 & 2.33 & 11.3 & 0.45 & 2.34 & 10.7 & 0.38 & 2.35 & 10.2 & 0.48 \\
\hline 2.81 & 13.3 & 0.47 & 2.83 & 12.4 & 0.47 & 2.83 & 12.4 & 0.53 & 2.84 & 11.9 & 0.50 \\
\hline 3.69 & 16.8 & 0.57 & 3.75 & 14.7 & 0.57 & 3.74 & 14.4 & 0.50 & 3.75 & 14.3 & 0.59 \\
\hline 4.68 & 18.2 & 0.73 & 4.70 & 17.4 & 0.73 & 4.72 & 16.5 & 0.71 & 4.71 & 16.7 & 0.68 \\
\hline
\end{tabular}


Table S5 5-HMF adsorption capacity on XAD761 resin in single-component system at various temperatures.

\begin{tabular}{|c|c|c|c|c|c|c|c|c|c|c|c|}
\hline \multicolumn{12}{|c|}{ Temperature } \\
\hline & $288 \mathrm{~K}$ & & & $298 \mathrm{~K}$ & & & $308 \mathrm{~K}$ & & & $318 \mathrm{~K}$ & \\
\hline $\begin{array}{c}c_{e} \\
(\mathrm{~g} / \mathrm{L})\end{array}$ & $\begin{array}{c}q_{e} \\
(\mathrm{mg} / \mathrm{g})\end{array}$ & STDV & $\begin{array}{c}c_{e} \\
(\mathrm{~g} / \mathrm{L})\end{array}$ & $\begin{array}{c}q_{e} \\
(\mathrm{mg} / \mathrm{g})\end{array}$ & STDV & $\begin{array}{c}c_{e} \\
(\mathrm{~g} / \mathrm{L})\end{array}$ & $\begin{array}{c}q_{e} \\
(\mathrm{mg} / \mathrm{g})\end{array}$ & STDV & $\begin{array}{c}c_{e} \\
(\mathrm{~g} / \mathrm{L})\end{array}$ & $\begin{array}{c}q_{e} \\
(\mathrm{mg} / \mathrm{g})\end{array}$ & STDV \\
\hline 0.00 & 0.0 & 0.00 & 0.00 & 0.0 & 0.00 & 0.00 & 0.0 & 0.00 & 0.00 & 0.0 & 0.00 \\
\hline 0.08 & 20.9 & 0.96 & 0.10 & 20.3 & 0.83 & 0.15 & 17.6 & 0.85 & 0.17 & 16.3 & 0.70 \\
\hline 0.30 & 36.8 & 1.58 & 0.35 & 34.4 & 1.55 & 0.41 & 31.3 & 1.44 & 0.45 & 29.8 & 1.13 \\
\hline 0.53 & 48.5 & 1.99 & 0.56 & 47.3 & 2.27 & 0.54 & 47.6 & 2.71 & 0.71 & 38.3 & 1.49 \\
\hline 0.84 & 58.6 & 2.29 & 0.89 & 57.2 & 2.46 & 1.04 & 49.8 & 1.89 & 1.10 & 46.4 & 1.81 \\
\hline 1.18 & 67.1 & 2.48 & 1.27 & 64.1 & 2.37 & 1.35 & 59.1 & 2.66 & 1.47 & 54.1 & 2.54 \\
\hline 1.55 & 74.4 & 3.42 & 1.63 & 71.3 & 2.50 & 1.73 & 64.9 & 2.85 & 1.84 & 62.0 & 2.85 \\
\hline 2.35 & 84.3 & 3.79 & 2.39 & 81.5 & 3.42 & 2.44 & 75.5 & 3.17 & 2.61 & 67.7 & 2.91 \\
\hline 3.09 & 92.2 & 3.87 & 3.26 & 89.5 & 3.67 & 3.32 & 84.9 & 3.74 & 3.40 & 78.7 & 3.23 \\
\hline
\end{tabular}


Table S6 LA adsorption capacity on XAD-4 resin in single-component system at various temperatures.

\begin{tabular}{|c|c|c|c|c|c|c|c|c|c|c|c|}
\hline \multicolumn{12}{|c|}{ Temperature } \\
\hline \multicolumn{3}{|c|}{$288 \mathrm{~K}$} & \multicolumn{3}{|c|}{$298 \mathrm{~K}$} & \multicolumn{3}{|c|}{$308 \mathrm{~K}$} & \multicolumn{3}{|c|}{$318 \mathrm{~K}$} \\
\hline $\begin{array}{c}c_{e} \\
(\mathrm{~g} / \mathrm{L})\end{array}$ & $\begin{array}{c}q_{e} \\
(\mathrm{mg} / \mathrm{g})\end{array}$ & STDV & $\begin{array}{c}c_{e} \\
(\mathrm{~g} / \mathrm{L})\end{array}$ & $\begin{array}{c}q_{e} \\
(\mathrm{mg} / \mathrm{g})\end{array}$ & STDV & $\begin{array}{c}c_{e} \\
(\mathrm{~g} / \mathrm{L})\end{array}$ & $\begin{array}{c}q_{e} \\
(\mathrm{mg} / \mathrm{g})\end{array}$ & STDV & $\begin{array}{c}c_{e} \\
(\mathrm{~g} / \mathrm{L})\end{array}$ & $\begin{array}{c}q_{e} \\
(\mathrm{mg} / \mathrm{g})\end{array}$ & STDV \\
\hline 0.00 & 0.0 & 0.00 & 0.00 & 0.0 & 0.00 & 0.00 & 0.0 & 0.00 & 0.00 & 0.0 & 0.00 \\
\hline 0.87 & 7.9 & 0.33 & 0.86 & 7.8 & 0.36 & 0.90 & 6.1 & 0.26 & 0.91 & 5.7 & 0.24 \\
\hline 2.31 & 14.6 & 0.67 & 2.32 & 13.7 & 0.66 & 2.35 & 12.0 & 0.54 & 2.37 & 10.6 & 0.39 \\
\hline 3.70 & 19.7 & 0.77 & 3.73 & 18.6 & 0.78 & 3.77 & 16.4 & 0.67 & 3.80 & 15.6 & 0.63 \\
\hline 5.15 & 24.3 & 1.02 & 5.17 & 23.9 & 1.07 & 5.22 & 21.4 & 0.83 & 5.25 & 19.7 & 0.83 \\
\hline 7.07 & 30.7 & 1.07 & 7.10 & 30.2 & 1.15 & 7.15 & 27.6 & 1.19 & 7.25 & 22.5 & 0.99 \\
\hline 9.53 & 36.0 & 1.55 & 9.60 & 34.1 & 1.26 & 9.58 & 35.0 & 1.47 & 9.68 & 29.6 & 1.33 \\
\hline 14.46 & 45.3 & 1.86 & 14.45 & 46.0 & 1.88 & 14.52 & 42.9 & 1.98 & 14.58 & 38.4 & 1.84 \\
\hline 19.29 & 55.0 & 2.15 & 19.30 & 53.1 & 2.12 & 19.38 & 51.0 & 2.19 & 19.50 & 45.3 & 1.90 \\
\hline
\end{tabular}


Table S7 LA adsorption capacity on XAD7HP resin in single-component system at various temperatures.

\begin{tabular}{|c|c|c|c|c|c|c|c|c|c|c|c|}
\hline \multicolumn{12}{|c|}{ Temperature } \\
\hline \multicolumn{3}{|c|}{$288 \mathrm{~K}$} & \multicolumn{3}{|c|}{$298 \mathrm{~K}$} & \multicolumn{3}{|c|}{$308 \mathrm{~K}$} & \multicolumn{3}{|c|}{$318 \mathrm{~K}$} \\
\hline $\begin{array}{c}c_{e} \\
(\mathrm{~g} / \mathrm{L})\end{array}$ & $\begin{array}{c}q_{e} \\
(\mathrm{mg} / \mathrm{g})\end{array}$ & STDV & $\begin{array}{c}c_{e} \\
(\mathrm{~g} / \mathrm{L})\end{array}$ & $\begin{array}{c}q_{e} \\
(\mathrm{mg} / \mathrm{g})\end{array}$ & STDV & $\begin{array}{c}c_{e} \\
(\mathrm{~g} / \mathrm{L})\end{array}$ & $\begin{array}{c}q_{e} \\
(\mathrm{mg} / \mathrm{g})\end{array}$ & STDV & $\begin{array}{c}c_{e} \\
(\mathrm{~g} / \mathrm{L})\end{array}$ & $\begin{array}{c}q_{e} \\
(\mathrm{mg} / \mathrm{g})\end{array}$ & STDV \\
\hline 0.00 & 0.0 & 0.00 & 0.00 & 0.0 & 0.00 & 0.00 & 0.0 & 0.00 & 0.00 & 0.0 & 0.00 \\
\hline 0.93 & 4.9 & 0.22 & 0.94 & 4.4 & 0.20 & 0.96 & 3.2 & 0.15 & 0.95 & 3.7 & 0.16 \\
\hline 2.42 & 8.6 & 0.29 & 2.43 & 8.4 & 0.36 & 2.44 & 7.9 & 0.34 & 2.47 & 6.3 & 0.26 \\
\hline 3.86 & 12.2 & 0.44 & 3.88 & 11.1 & 0.48 & 3.87 & 11.3 & 0.52 & 3.90 & 10.2 & 0.46 \\
\hline 5.34 & 15.8 & 0.71 & 5.33 & 15.9 & 0.77 & 5.32 & 15.8 & 0.66 & 5.37 & 13.9 & 0.68 \\
\hline 7.24 & 22.9 & 1.10 & 7.29 & 20.5 & 0.96 & 7.32 & 19.4 & 0.76 & 7.36 & 15.9 & 0.67 \\
\hline 9.71 & 28.8 & 1.24 & 9.76 & 26.0 & 1.27 & 9.79 & 24.9 & 0.92 & 9.86 & 21.5 & 1.01 \\
\hline 14.62 & 37.0 & 1.55 & 14.62 & 33.7 & 1.41 & 14.73 & 30.2 & 1.36 & 14.82 & 27.4 & 1.32 \\
\hline 19.54 & 44.2 & 1.99 & 19.62 & 39.9 & 1.64 & 19.67 & 37.2 & 1.75 & 19.72 & 34.7 & 1.42 \\
\hline
\end{tabular}


Table S8 LA adsorption capacity on XAD761 resin in single-component system at various temperatures.

\begin{tabular}{|c|c|c|c|c|c|c|c|c|c|c|c|}
\hline \multicolumn{12}{|c|}{ Temperature } \\
\hline \multicolumn{3}{|c|}{$288 \mathrm{~K}$} & \multicolumn{3}{|c|}{$298 \mathrm{~K}$} & \multicolumn{3}{|c|}{$308 \mathrm{~K}$} & \multicolumn{3}{|c|}{$318 \mathrm{~K}$} \\
\hline $\begin{array}{c}c_{e} \\
(\mathrm{~g} / \mathrm{L})\end{array}$ & $\begin{array}{c}q_{e} \\
(\mathrm{mg} / \mathrm{g})\end{array}$ & STDV & $\begin{array}{c}c_{e} \\
(\mathrm{~g} / \mathrm{L})\end{array}$ & $\begin{array}{c}q_{e} \\
(\mathrm{mg} / \mathrm{g})\end{array}$ & STDV & $\begin{array}{c}c_{e} \\
(\mathrm{~g} / \mathrm{L})\end{array}$ & $\begin{array}{c}q_{e} \\
(\mathrm{mg} / \mathrm{g})\end{array}$ & STDV & $\begin{array}{c}c_{e} \\
(\mathrm{~g} / \mathrm{L})\end{array}$ & $\begin{array}{c}q_{e} \\
(\mathrm{mg} / \mathrm{g})\end{array}$ & STDV \\
\hline 0.00 & 0.0 & 0.00 & 0.00 & 0.0 & 0.00 & 0.00 & 0.0 & 0.00 & 0.00 & 0.0 & 0.00 \\
\hline 0.51 & 24.9 & 1.04 & 0.58 & 21.7 & 0.93 & 0.61 & 20.6 & 0.80 & 0.65 & 18.3 & 0.75 \\
\hline 1.68 & 44.8 & 2.01 & 1.74 & 41.4 & 1.70 & 1.80 & 39.0 & 1.64 & 1.89 & 34.2 & 1.30 \\
\hline 2.91 & 57.7 & 2.48 & 3.02 & 53.5 & 2.19 & 3.07 & 50.1 & 2.20 & 3.15 & 47.4 & 1.85 \\
\hline 4.26 & 67.5 & 2.77 & 4.35 & 65.2 & 2.93 & 4.40 & 61.4 & 2.52 & 4.50 & 56.9 & 2.45 \\
\hline 6.10 & 80.3 & 3.45 & 6.19 & 75.7 & 3.25 & 6.24 & 71.4 & 3.00 & 6.42 & 64.2 & 2.95 \\
\hline 8.48 & 89.7 & 3.50 & 8.44 & 87.3 & 3.67 & 8.61 & 83.0 & 3.90 & 8.75 & 74.9 & 3.52 \\
\hline 13.24 & 104.5 & 3.97 & 13.32 & 101.4 & 4.56 & 13.38 & 98.0 & 4.41 & 13.44 & 87.9 & 3.60 \\
\hline 18.06 & 117.4 & 4.11 & 18.16 & 112.6 & 5.18 & 18.19 & 109.0 & 4.69 & 18.27 & 103.3 & 3.82 \\
\hline
\end{tabular}


Table S9 FA adsorption capacity on XAD-4 resin in single-component system at various temperatures.

\begin{tabular}{|c|c|c|c|c|c|c|c|c|c|c|c|}
\hline \multicolumn{12}{|c|}{ Temperature } \\
\hline & $288 \mathrm{~K}$ & & & $298 \mathrm{~K}$ & & & $308 \mathrm{~K}$ & & & $318 \mathrm{~K}$ & \\
\hline $\begin{array}{c}c_{e} \\
(\mathrm{~g} / \mathrm{L})\end{array}$ & $\begin{array}{c}q_{e} \\
(\mathrm{mg} / \mathrm{g})\end{array}$ & STDV & $\begin{array}{c}c_{e} \\
(\mathrm{~g} / \mathrm{L})\end{array}$ & $\begin{array}{c}q_{e} \\
(\mathrm{mg} / \mathrm{g})\end{array}$ & STDV & $\begin{array}{c}c_{e} \\
(\mathrm{~g} / \mathrm{L})\end{array}$ & $\begin{array}{c}q_{e} \\
(\mathrm{mg} / \mathrm{g})\end{array}$ & STDV & $\begin{array}{c}c_{e} \\
(\mathrm{~g} / \mathrm{L})\end{array}$ & $\begin{array}{c}q_{e} \\
(\mathrm{mg} / \mathrm{g})\end{array}$ & STDV \\
\hline 0.00 & 0.0 & 0.00 & 0.00 & 0.0 & 0.00 & 0.00 & 0.0 & 0.00 & 0.00 & 0.0 & 0.00 \\
\hline 0.94 & 0.7 & 0.03 & 0.93 & 1.0 & 0.05 & 0.93 & 0.8 & 0.04 & 0.94 & 0.4 & 0.02 \\
\hline 1.80 & 2.0 & 0.08 & 1.79 & 2.4 & 0.11 & 1.79 & 2.3 & 0.10 & 1.80 & 2.0 & 0.09 \\
\hline 2.75 & 3.0 & 0.14 & 2.75 & 3.0 & 0.14 & 2.74 & 3.5 & 0.15 & 2.76 & 2.6 & 0.13 \\
\hline 3.64 & 4.1 & 0.18 & 3.63 & 4.6 & 0.20 & 3.64 & 3.9 & 0.16 & 3.65 & 3.5 & 0.15 \\
\hline 5.09 & 5.5 & 0.25 & 5.08 & 6.0 & 0.24 & 5.09 & 5.9 & 0.28 & 5.11 & 4.7 & 0.20 \\
\hline 6.32 & 6.6 & 0.28 & 6.33 & 6.5 & 0.27 & 6.32 & 6.9 & 0.33 & 6.35 & 5.6 & 0.25 \\
\hline 7.65 & 7.5 & 0.34 & 7.64 & 8.4 & 0.38 & 7.65 & 8.3 & 0.35 & 7.68 & 6.6 & 0.28 \\
\hline 8.95 & 8.6 & 0.37 & 8.93 & 9.3 & 0.43 & 8.95 & 8.6 & 0.42 & 8.97 & 7.3 & 0.31 \\
\hline
\end{tabular}


Table S10 FA adsorption capacity on XAD7HP resin in single-component system at various temperatures.

\begin{tabular}{|c|c|c|c|c|c|c|c|c|c|c|c|}
\hline \multicolumn{12}{|c|}{ Temperature } \\
\hline \multicolumn{3}{|c|}{$288 \mathrm{~K}$} & \multicolumn{3}{|c|}{$298 \mathrm{~K}$} & \multicolumn{3}{|c|}{$308 \mathrm{~K}$} & \multicolumn{3}{|c|}{$318 \mathrm{~K}$} \\
\hline $\begin{array}{c}c_{e} \\
(\mathrm{~g} / \mathrm{L})\end{array}$ & $\begin{array}{c}q_{e} \\
(\mathrm{mg} / \mathrm{g})\end{array}$ & STDV & $\begin{array}{c}c_{e} \\
(\mathrm{~g} / \mathrm{L})\end{array}$ & $\begin{array}{c}q_{e} \\
(\mathrm{mg} / \mathrm{g})\end{array}$ & STDV & $\begin{array}{c}c_{e} \\
(\mathrm{~g} / \mathrm{L})\end{array}$ & $\begin{array}{c}q_{e} \\
(\mathrm{mg} / \mathrm{g})\end{array}$ & STDV & $\begin{array}{c}c_{e} \\
(\mathrm{~g} / \mathrm{L})\end{array}$ & $\begin{array}{c}q_{e} \\
(\mathrm{mg} / \mathrm{g})\end{array}$ & STDV \\
\hline 0.00 & 0.0 & 0.00 & 0.00 & 0.0 & 0.00 & 0.00 & 0.0 & 0.00 & 0.00 & 0.0 & 0.00 \\
\hline 0.94 & 0.8 & 0.04 & 0.92 & 1.6 & 0.08 & 0.92 & 1.5 & 0.07 & 0.92 & 1.4 & 0.06 \\
\hline 1.80 & 1.7 & 0.08 & 1.79 & 2.4 & 0.11 & 1.79 & 2.1 & 0.10 & 1.80 & 2.0 & 0.09 \\
\hline 2.76 & 2.4 & 0.12 & 2.75 & 3.0 & 0.13 & 2.75 & 2.9 & 0.14 & 2.76 & 2.3 & 0.10 \\
\hline 3.64 & 3.7 & 0.18 & 3.65 & 3.6 & 0.16 & 3.67 & 2.6 & 0.12 & 3.66 & 3.2 & 0.15 \\
\hline 5.10 & 5.0 & 0.23 & 5.10 & 5.2 & 0.23 & 5.11 & 4.4 & 0.21 & 5.12 & 4.3 & 0.21 \\
\hline 6.33 & 6.4 & 0.29 & 6.35 & 5.3 & 0.25 & 6.37 & 4.6 & 0.22 & 6.36 & 5.1 & 0.25 \\
\hline 7.67 & 7.0 & 0.34 & 7.68 & 6.5 & 0.30 & 7.67 & 6.4 & 0.31 & 7.68 & 6.4 & 0.31 \\
\hline 8.96 & 8.1 & 0.38 & 8.97 & 7.8 & 0.37 & 8.96 & 7.4 & 0.33 & 8.98 & 7.2 & 0.33 \\
\hline
\end{tabular}


Table S11 FA adsorption capacity on XAD761 resin in single-component system at various temperatures.

\begin{tabular}{|c|c|c|c|c|c|c|c|c|c|c|c|}
\hline \multicolumn{12}{|c|}{ Temperature } \\
\hline \multicolumn{3}{|c|}{$288 \mathrm{~K}$} & \multicolumn{3}{|c|}{$298 \mathrm{~K}$} & \multicolumn{3}{|c|}{$308 \mathrm{~K}$} & \multicolumn{3}{|c|}{$318 \mathrm{~K}$} \\
\hline $\begin{array}{c}c_{e} \\
(\mathrm{~g} / \mathrm{L})\end{array}$ & $\begin{array}{c}q_{e} \\
(\mathrm{mg} / \mathrm{g})\end{array}$ & STDV & $\begin{array}{c}c_{e} \\
(\mathrm{~g} / \mathrm{L})\end{array}$ & $\begin{array}{c}q_{e} \\
(\mathrm{mg} / \mathrm{g})\end{array}$ & STDV & $\begin{array}{c}c_{e} \\
(\mathrm{~g} / \mathrm{L})\end{array}$ & $\begin{array}{c}q_{e} \\
(\mathrm{mg} / \mathrm{g})\end{array}$ & STDV & $\begin{array}{c}c_{e} \\
(\mathrm{~g} / \mathrm{L})\end{array}$ & $\begin{array}{c}q_{e} \\
(\mathrm{mg} / \mathrm{g})\end{array}$ & STDV \\
\hline 0.00 & 0.0 & 0.00 & 0.00 & 0.0 & 0.00 & 0.00 & 0.0 & 0.00 & 0.00 & 0.0 & 0.00 \\
\hline 0.92 & 1.8 & 0.08 & 0.91 & 2.2 & 0.10 & 0.91 & 1.8 & 0.08 & 0.92 & 1.4 & 0.06 \\
\hline 1.76 & 4.0 & 0.19 & 1.77 & 3.3 & 0.15 & 1.76 & 3.9 & 0.18 & 1.79 & 2.3 & 0.10 \\
\hline 2.70 & 5.5 & 0.27 & 2.70 & 5.3 & 0.24 & 2.71 & 4.9 & 0.23 & 2.73 & 3.9 & 0.16 \\
\hline 3.57 & 7.1 & 0.31 & 3.58 & 7.1 & 0.32 & 3.59 & 6.3 & 0.27 & 3.61 & 5.2 & 0.21 \\
\hline 5.01 & 9.4 & 0.42 & 5.01 & 9.2 & 0.38 & 5.03 & 8.6 & 0.35 & 5.04 & 7.9 & 0.36 \\
\hline 6.20 & 11.9 & 0.55 & 6.23 & 11.2 & 0.47 & 6.23 & 10.7 & 0.45 & 6.25 & 9.9 & 0.48 \\
\hline 7.53 & 13.8 & 0.58 & 7.54 & 13.0 & 0.52 & 7.55 & 12.8 & 0.55 & 7.59 & 10.9 & 0.47 \\
\hline 8.81 & 15.9 & 0.73 & 8.82 & 15.1 & 0.71 & 8.81 & 14.6 & 0.70 & 8.85 & 12.6 & 0.58 \\
\hline
\end{tabular}


Table S12 Glucose adsorption capacity on XAD-4 resin in single-component system at various temperatures.

\begin{tabular}{|c|c|c|c|c|c|c|c|c|c|c|c|}
\hline \multicolumn{12}{|c|}{ Temperature } \\
\hline \multicolumn{3}{|c|}{$288 \mathrm{~K}$} & \multicolumn{3}{|c|}{$298 \mathrm{~K}$} & \multicolumn{3}{|c|}{$308 \mathrm{~K}$} & \multicolumn{3}{|c|}{$318 \mathrm{~K}$} \\
\hline $\begin{array}{c}c_{e} \\
(\mathrm{~g} / \mathrm{L})\end{array}$ & $\begin{array}{c}q_{e} \\
(\mathrm{mg} / \mathrm{g})\end{array}$ & STDV & $\begin{array}{c}c_{e} \\
(\mathrm{~g} / \mathrm{L})\end{array}$ & $\begin{array}{c}q_{e} \\
(\mathrm{mg} / \mathrm{g})\end{array}$ & STDV & $\begin{array}{c}c_{e} \\
(\mathrm{~g} / \mathrm{L})\end{array}$ & $\begin{array}{c}q_{e} \\
(\mathrm{mg} / \mathrm{g})\end{array}$ & STDV & $\begin{array}{c}c_{e} \\
(\mathrm{~g} / \mathrm{L})\end{array}$ & $\begin{array}{c}q_{e} \\
(\mathrm{mg} / \mathrm{g})\end{array}$ & STDV \\
\hline 0.00 & 0.0 & 0.00 & 0.00 & 0.0 & 0.00 & 0.00 & 0.0 & 0.00 & 0.00 & 0.0 & 0.00 \\
\hline 1.01 & 0.6 & 0.03 & 1.01 & 0.4 & 0.02 & 1.01 & 0.3 & 0.01 & 1.02 & 0.1 & 0.00 \\
\hline 1.90 & 0.9 & 0.03 & 1.91 & 0.7 & 0.03 & 1.90 & 0.9 & 0.03 & 1.91 & 0.6 & 0.02 \\
\hline 2.85 & 1.2 & 0.05 & 2.84 & 1.4 & 0.06 & 2.84 & 1.5 & 0.06 & 2.85 & 1.1 & 0.05 \\
\hline 3.79 & 2.9 & 0.12 & 3.80 & 2.6 & 0.11 & 3.80 & 2.5 & 0.12 & 3.81 & 2.1 & 0.09 \\
\hline 5.13 & 4.3 & 0.19 & 5.15 & 3.5 & 0.16 & 5.16 & 3.2 & 0.14 & 5.17 & 2.6 & 0.12 \\
\hline 6.70 & 4.9 & 0.20 & 6.71 & 4.4 & 0.17 & 6.71 & 4.0 & 0.17 & 6.73 & 3.4 & 0.15 \\
\hline 7.93 & 6.0 & 0.23 & 7.94 & 5.3 & 0.23 & 7.97 & 4.5 & 0.18 & 7.98 & 4.0 & 0.17 \\
\hline 9.39 & 6.7 & 0.27 & 9.40 & 6.2 & 0.28 & 9.41 & 6.0 & 0.26 & 9.43 & 4.8 & 0.20 \\
\hline
\end{tabular}


Table S13 Glucose adsorption capacity on XAD7HP resin in single-component system at various temperatures.

\begin{tabular}{|c|c|c|c|c|c|c|c|c|c|c|c|}
\hline \multicolumn{12}{|c|}{ Temperature } \\
\hline \multicolumn{3}{|c|}{$288 \mathrm{~K}$} & \multicolumn{3}{|c|}{$298 \mathrm{~K}$} & \multicolumn{3}{|c|}{$308 \mathrm{~K}$} & \multicolumn{3}{|c|}{$318 \mathrm{~K}$} \\
\hline $\begin{array}{c}c_{e} \\
(\mathrm{~g} / \mathrm{L})\end{array}$ & $\begin{array}{c}q_{e} \\
(\mathrm{mg} / \mathrm{g})\end{array}$ & STDV & $\begin{array}{c}c_{e} \\
(\mathrm{~g} / \mathrm{L})\end{array}$ & $\begin{array}{c}q_{e} \\
(\mathrm{mg} / \mathrm{g})\end{array}$ & STDV & $\begin{array}{c}c_{e} \\
(\mathrm{~g} / \mathrm{L})\end{array}$ & $\begin{array}{c}q_{e} \\
(\mathrm{mg} / \mathrm{g})\end{array}$ & STDV & $\begin{array}{c}c_{e} \\
(\mathrm{~g} / \mathrm{L})\end{array}$ & $\begin{array}{c}q_{e} \\
(\mathrm{mg} / \mathrm{g})\end{array}$ & STDV \\
\hline 0.00 & 0.0 & 0.00 & 0.00 & 0.0 & 0.00 & 0.00 & 0.0 & 0.00 & 0.00 & 0.0 & 0.00 \\
\hline 1.01 & 0.4 & 0.02 & 1.01 & 0.4 & 0.02 & 1.01 & 0.2 & 0.01 & 1.02 & 0.0 & 0.00 \\
\hline 1.90 & 1.0 & 0.05 & 1.90 & 0.9 & 0.04 & 1.91 & 0.5 & 0.02 & 1.91 & 0.5 & 0.02 \\
\hline 2.83 & 1.8 & 0.09 & 2.84 & 1.6 & 0.07 & 2.85 & 1.1 & 0.05 & 2.84 & 1.3 & 0.07 \\
\hline 3.79 & 2.7 & 0.13 & 3.80 & 2.6 & 0.12 & 3.80 & 2.3 & 0.10 & 3.81 & 2.0 & 0.09 \\
\hline 5.15 & 3.5 & 0.16 & 5.15 & 3.3 & 0.16 & 5.16 & 2.9 & 0.12 & 5.17 & 2.5 & 0.10 \\
\hline 6.70 & 4.6 & 0.21 & 6.70 & 4.7 & 0.20 & 6.71 & 4.1 & 0.19 & 6.72 & 3.3 & 0.13 \\
\hline 7.94 & 5.4 & 0.23 & 7.94 & 5.5 & 0.23 & 7.95 & 4.7 & 0.20 & 7.97 & 4.1 & 0.17 \\
\hline 9.38 & 6.8 & 0.28 & 9.41 & 5.9 & 0.25 & 9.42 & 5.1 & 0.21 & 9.43 & 4.8 & 0.21 \\
\hline
\end{tabular}


Table S14 Glucose adsorption capacity on XAD761 resin in single-component system at various temperatures.

\begin{tabular}{|c|c|c|c|c|c|c|c|c|c|c|c|}
\hline \multicolumn{12}{|c|}{ Temperature } \\
\hline \multicolumn{3}{|c|}{$288 \mathrm{~K}$} & \multicolumn{3}{|c|}{$298 \mathrm{~K}$} & \multicolumn{3}{|c|}{$308 \mathrm{~K}$} & \multicolumn{3}{|c|}{$318 \mathrm{~K}$} \\
\hline $\begin{array}{c}c_{e} \\
(\mathrm{~g} / \mathrm{L})\end{array}$ & $\begin{array}{c}q_{e} \\
(\mathrm{mg} / \mathrm{g})\end{array}$ & STDV & $\begin{array}{c}c_{e} \\
(\mathrm{~g} / \mathrm{L})\end{array}$ & $\begin{array}{c}q_{e} \\
(\mathrm{mg} / \mathrm{g})\end{array}$ & STDV & $\begin{array}{c}c_{e} \\
(\mathrm{~g} / \mathrm{L})\end{array}$ & $\begin{array}{c}q_{e} \\
(\mathrm{mg} / \mathrm{g})\end{array}$ & STDV & $\begin{array}{c}c_{e} \\
(\mathrm{~g} / \mathrm{L})\end{array}$ & $\begin{array}{c}q_{e} \\
(\mathrm{mg} / \mathrm{g})\end{array}$ & STDV \\
\hline 0.00 & 0.0 & 0.00 & 0.00 & 0.0 & 0.00 & 0.00 & 0.0 & 0.00 & 0.00 & 0.0 & 0.00 \\
\hline 1.01 & 0.1 & 0.01 & 1.02 & 0.1 & 0.00 & 1.01 & 0.1 & 0.01 & 1.02 & 0.0 & 0.00 \\
\hline 1.92 & 0.2 & 0.01 & 1.92 & 0.3 & 0.01 & 1.92 & 0.2 & 0.01 & 1.92 & 0.1 & 0.01 \\
\hline 2.86 & 0.5 & 0.02 & 2.86 & 0.6 & 0.03 & 2.87 & 0.3 & 0.01 & 2.87 & 0.2 & 0.01 \\
\hline 3.84 & 0.7 & 0.03 & 3.84 & 0.8 & 0.03 & 3.84 & 0.4 & 0.02 & 3.84 & 0.4 & 0.01 \\
\hline 5.20 & 1.2 & 0.05 & 5.21 & 0.9 & 0.04 & 5.21 & 0.9 & 0.03 & 5.21 & 0.7 & 0.03 \\
\hline 6.77 & 1.4 & 0.07 & 6.77 & 1.2 & 0.05 & 6.77 & 1.2 & 0.05 & 6.78 & 0.9 & 0.03 \\
\hline 8.02 & 2.1 & 0.08 & 8.02 & 1.6 & 0.06 & 8.03 & 1.5 & 0.07 & 8.03 & 1.4 & 0.06 \\
\hline 9.47 & 2.6 & 0.11 & 9.48 & 2.1 & 0.09 & 9.49 & 1.9 & 0.08 & 9.50 & 1.6 & 0.07 \\
\hline
\end{tabular}


Table S15 The effect of temperature on the adsorption rate of 5-HMF adsorbed onto XAD761 resin at initial concentration $C_{0}=2.71 \mathrm{~g} \mathrm{~L}^{-1}$ and resin dosage $m=20 \mathrm{~g} \mathrm{~L}^{-1}$.

\begin{tabular}{|c|c|c|c|c|c|c|c|c|}
\hline \multirow{3}{*}{$\begin{array}{c}t \\
(\min )\end{array}$} & \multicolumn{8}{|c|}{ Temperature } \\
\hline & \multicolumn{2}{|c|}{$288 \mathrm{~K}$} & \multicolumn{2}{|c|}{$298 \mathrm{~K}$} & \multicolumn{2}{|c|}{$308 \mathrm{~K}$} & \multicolumn{2}{|c|}{$318 \mathrm{~K}$} \\
\hline & $\begin{array}{c}q_{t} \\
(\mathrm{mg} / \mathrm{g})\end{array}$ & STDV & $\begin{array}{c}q_{t} \\
(\mathrm{mg} / \mathrm{g})\end{array}$ & STDV & $\begin{array}{c}q_{t} \\
(\mathrm{mg} / \mathrm{g})\end{array}$ & STDV & $\begin{array}{c}q_{t} \\
(\mathrm{mg} / \mathrm{g})\end{array}$ & STDV \\
\hline 0 & 0.0 & 0.00 & 0.0 & 0.00 & 0.0 & 0.00 & 0.0 & 0.00 \\
\hline 0.5 & 8.6 & 0.22 & 13.8 & 0.32 & 11.9 & 0.31 & 14.6 & 0.35 \\
\hline 1 & 12.9 & 0.30 & 16.1 & 0.39 & 14.9 & 0.40 & 18.4 & 0.28 \\
\hline 2 & 16.6 & 0.45 & 20.2 & 0.59 & 20.3 & 0.32 & 23.7 & 0.64 \\
\hline 3 & 20.0 & 0.44 & 23.4 & 0.63 & 24.2 & 0.43 & 27.9 & 0.53 \\
\hline 5 & 25.0 & 0.53 & 28.6 & 0.51 & 29.6 & 0.56 & 33.7 & 0.74 \\
\hline 10 & 33.9 & 0.78 & 37.2 & 0.71 & 38.3 & 0.80 & 42.3 & 0.72 \\
\hline 20 & 44.3 & 1.15 & 46.8 & 0.80 & 47.8 & 0.81 & 50.5 & 0.91 \\
\hline 30 & 50.7 & 1.32 & 52.6 & 0.79 & 52.4 & 1.15 & 53.9 & 1.40 \\
\hline 60 & 61.0 & 1.71 & 60.7 & 1.40 & 57.1 & 1.43 & 56.9 & 1.31 \\
\hline 120 & 68.4 & 1.44 & 64.3 & 1.61 & 58.9 & 1.65 & 57.5 & 1.44 \\
\hline 180 & 70.3 & 1.27 & 65.0 & 1.43 & 59.0 & 1.53 & 57.5 & 1.27 \\
\hline 240 & 70.9 & 1.35 & 65.1 & 1.56 & 59.0 & 1.42 & 57.5 & 1.44 \\
\hline
\end{tabular}


Table S16 Pseudo-first-order kinetic models for the adsorption of 5-HMF onto XAD761 resin at various temperatures.

\begin{tabular}{|c|c|c|c|c|c|c|c|c|}
\hline \multirow{3}{*}{$\begin{array}{c}t \\
(\min )\end{array}$} & \multicolumn{8}{|c|}{ Temperature } \\
\hline & \multicolumn{2}{|c|}{$288 \mathrm{~K}$} & \multicolumn{2}{|c|}{$298 \mathrm{~K}$} & \multicolumn{2}{|c|}{$308 \mathrm{~K}$} & \multicolumn{2}{|c|}{$318 \mathrm{~K}$} \\
\hline & $\ln \left(q_{e^{-}} q_{t}\right)$ & STDV & $\ln \left(q_{e^{-}} q_{t}\right)$ & STDV & $\ln \left(q_{e^{-}} q_{t}\right)$ & STDV & $\ln \left(q_{e^{-}} q_{t}\right)$ & STDV \\
\hline 0 & 4.262 & 0.136 & 4.176 & 0.146 & 4.078 & 0.163 & 4.052 & 0.154 \\
\hline 0.5 & 4.132 & 0.165 & 3.939 & 0.165 & 3.853 & 0.166 & 3.759 & 0.180 \\
\hline 1 & 4.060 & 0.183 & 3.891 & 0.160 & 3.787 & 0.106 & 3.668 & 0.172 \\
\hline 2 & 3.996 & 0.152 & 3.804 & 0.167 & 3.656 & 0.154 & 3.521 & 0.144 \\
\hline 3 & 3.931 & 0.169 & 3.731 & 0.142 & 3.551 & 0.163 & 3.388 & 0.142 \\
\hline 5 & 3.827 & 0.138 & 3.599 & 0.162 & 3.381 & 0.128 & 3.171 & 0.136 \\
\hline 10 & 3.612 & 0.170 & 3.328 & 0.143 & 3.032 & 0.109 & 2.724 & 0.106 \\
\hline 20 & 3.282 & 0.138 & 2.905 & 0.113 & 2.419 & 0.109 & 1.955 & 0.070 \\
\hline 30 & 3.006 & 0.120 & 2.527 & 0.104 & 1.891 & 0.079 & 1.302 & 0.053 \\
\hline 60 & 2.297 & 0.106 & 1.494 & 0.055 & 0.624 & 0.026 & -0.397 & 0.017 \\
\hline 120 & 0.924 & 0.040 & -0.222 & 0.010 & -2.216 & 0.086 & -3.919 & 0.176 \\
\hline 180 & -0.435 & 0.017 & -1.793 & 0.079 & & & & \\
\hline
\end{tabular}


Table S17 Pseudo-second-order kinetic models for the adsorption of 5-HMF onto XAD761 resin at various temperatures.

\begin{tabular}{|c|c|c|c|c|c|c|c|c|}
\hline \multirow{3}{*}{$\begin{array}{c}t \\
(\min )\end{array}$} & \multicolumn{8}{|c|}{ Temperature } \\
\hline & \multicolumn{2}{|c|}{$288 \mathrm{~K}$} & \multicolumn{2}{|c|}{$298 \mathrm{~K}$} & \multicolumn{2}{|c|}{$308 \mathrm{~K}$} & \multicolumn{2}{|c|}{$318 \mathrm{~K}$} \\
\hline & $t / q_{t}$ & STDV & $t / q_{t}$ & STDV & $t / q_{t}$ & STDV & $t / q_{t}$ & STDV \\
\hline 0.5 & 0.058 & 0.025 & 0.036 & 0.001 & 0.042 & 0.002 & 0.034 & 0.002 \\
\hline 1 & 0.077 & 0.004 & 0.062 & 0.002 & 0.067 & 0.003 & 0.054 & 0.003 \\
\hline 2 & 0.121 & 0.004 & 0.099 & 0.004 & 0.098 & 0.004 & 0.084 & 0.004 \\
\hline 3 & 0.150 & 0.006 & 0.128 & 0.005 & 0.124 & 0.005 & 0.107 & 0.005 \\
\hline 5 & 0.200 & 0.009 & 0.175 & 0.008 & 0.169 & 0.006 & 0.148 & 0.007 \\
\hline 10 & 0.295 & 0.010 & 0.269 & 0.012 & 0.261 & 0.011 & 0.236 & 0.011 \\
\hline 20 & 0.451 & 0.020 & 0.427 & 0.019 & 0.419 & 0.018 & 0.396 & 0.017 \\
\hline 30 & 0.591 & 0.024 & 0.570 & 0.022 & 0.573 & 0.022 & 0.557 & 0.026 \\
\hline 60 & 0.984 & 0.042 & 0.989 & 0.038 & 1.050 & 0.040 & 1.055 & 0.044 \\
\hline 120 & 1.754 & 0.075 & 1.866 & 0.078 & 2.038 & 0.084 & 2.087 & 0.081 \\
\hline 180 & 2.561 & 0.100 & 2.771 & 0.114 & 3.051 & 0.131 & 3.130 & 0.116 \\
\hline 240 & 3.383 & 0.118 & 3.686 & 0.173 & 4.068 & 0.171 & 4.172 & 0.188 \\
\hline
\end{tabular}


Table S18 Intraparticle diffusion kinetic models for the adsorption of 5-HMF onto XAD761 resin at various temperatures.

\begin{tabular}{|c|c|c|c|c|c|c|c|c|}
\hline \multirow{3}{*}{$t^{0.5}$} & \multicolumn{8}{|c|}{ Temperature } \\
\hline & \multicolumn{2}{|c|}{$288 \mathrm{~K}$} & \multicolumn{2}{|c|}{$298 \mathrm{~K}$} & \multicolumn{2}{|c|}{$308 \mathrm{~K}$} & \multicolumn{2}{|c|}{$318 \mathrm{~K}$} \\
\hline & $\begin{array}{c}q_{t} \\
(\mathrm{mg} / \mathrm{g})\end{array}$ & STDV & $\begin{array}{c}q_{t} \\
(\mathrm{mg} / \mathrm{g})\end{array}$ & STDV & $\begin{array}{c}q_{t} \\
(\mathrm{mg} / \mathrm{g})\end{array}$ & STDV & $\begin{array}{c}q_{t} \\
(\mathrm{mg} / \mathrm{g})\end{array}$ & STDV \\
\hline 0.71 & 8.6 & 0.37 & 13.8 & 0.62 & 11.9 & 0.50 & 14.6 & 0.63 \\
\hline 1.00 & 12.9 & 0.60 & 16.1 & 0.69 & 14.9 & 0.69 & 18.4 & 0.75 \\
\hline 1.41 & 16.6 & 0.65 & 20.2 & 0.97 & 20.3 & 0.79 & 23.7 & 1.12 \\
\hline 1.73 & 20.0 & 0.88 & 23.4 & 0.91 & 24.2 & 0.99 & 27.9 & 1.23 \\
\hline 2.24 & 25.0 & 1.13 & 28.6 & 1.06 & 29.6 & 1.15 & 33.7 & 1.31 \\
\hline 3.16 & 33.9 & 1.29 & 37.2 & 1.71 & 38.3 & 1.68 & 42.3 & 1.56 \\
\hline 4.47 & 44.3 & 1.86 & 46.8 & 2.11 & 47.8 & 2.01 & 50.5 & 1.77 \\
\hline 5.48 & 50.7 & 2.38 & 52.6 & 2.26 & 52.4 & 2.10 & 53.9 & 2.21 \\
\hline 7.75 & 61.0 & 2.81 & 60.7 & 2.55 & 57.1 & 2.46 & 56.9 & 2.44 \\
\hline 10.95 & 68.4 & 3.08 & 64.3 & 3.02 & 58.9 & 2.41 & 57.5 & 2.53 \\
\hline 13.42 & 70.3 & 3.23 & 65.0 & 2.79 & 59.0 & 2.71 & 57.5 & 2.42 \\
\hline 15.49 & 70.9 & 3.05 & 65.1 & 2.93 & 59.0 & 2.66 & 57.5 & 2.36 \\
\hline
\end{tabular}


Table S19 Plot of Arrhenius equation for the adsorption of 5-HMF onto XAD761 resin.

\begin{tabular}{ccc}
\hline $1 / T$ & $\ln k_{2}$ & STDV \\
\hline 0.0035 & -6.5023 & 0.0226 \\
0.0034 & -5.9522 & 0.0200 \\
0.0032 & -5.4968 & 0.0178 \\
0.0031 & -5.0515 & 0.0159 \\
\hline
\end{tabular}

